УДК 62-531.32

\title{
ОПТИМИЗАЦИЯ ПРОЦЕССА ЗАКАЧКИ ФИКСИРОВАННЫХ ОБЪЕМОВ ЖИДКОСТИ В ДВА НАПРАВЛЕНИЯ
}

\section{Бикбулатова Голия Ильдусовна',} agni-ngo@mail.ru

Галеев Ахметсалим Сабирович', agni-ngo@mail.ru

Болтнева Юлия Анатольевна', agni-ngo@mail.ru

Ларин Пётр Андреевич², kafedra.itmen@mail.ru

\section{Сулейманов Раис Насибович²,} kafedra.itmen@mail.ru

\author{
Филимонов Олег Владимирович², \\ kafedra.itmen@mail.ru \\ Альметьевский государственный нефтяной институт, \\ Россия, 423458, Республика Татарстан, г. Альметьевск, ул. Ленина, 2. \\ 2 Филиал Уфимского государственного нефтяного технического университета в г. Октябрьском, \\ Россия, Республика Башкортостан, 452620, г. Октябрьский, ул. Девонская, 54 а.
}

Представлен новый подход к решению задачи оптимизации работы динамической системы «насос+трубопроводная сеть». В статье состоянием процесса закачки называются объёмы жидкости, поступившие через каждый трубопровод в данный момент времени. Эти объёмы взяты в качестве основных функций, зависящих от времени и описывающих процесс закачки. Система функций определяет воображаемую линию - траекторию закачки в пространстве, задаваемом плановыми объёмами закачки через каждый трубопровод. Регулирование потока в трубопроводах любыми устройствами, создающими местное гидродинамическое сопротивление (устройствами) даёт возможность получать различные траектории. Влияние регулирующих устройств оценивается коэффициентом открытости трубопровода или потока, определяемым как отношение потока (расхода) в частично перекрытом трубопроводе к потоку в полностью открытом трубопроводе. Рассмотрены два варианта закачки, в которых продемонстрировано, что разным траекториям может соответствовать Своя потребляемая энергия. Проведён анализ используемой при количественных исследованиях и оценке работы насоса величины, равной отношению напора, создаваемого насосом, к КПД, соответствующему этому напору. Показано, что при повышении напора или снижении подачи данная величина возрастает. Поскольку затрачиваемая насосом энергия зависит от формы траектории, энергия становится функционалом, для поиска минимума которого привлекаются методы вариационного исчисления. Таким способом находится экстремаль - траектория, на которой затраты энергии и времени минимальны. Траекторией, описывающей оптимальный режим закачки, оказывается прямолинейный отрезок в пространстве закачки, концы которого соответствуют начальному и конечному состояниям процесса закачки. Для реализации данного процесса предложен алгоритм, позволяющий определить, какой трубопровод следует частично перекрыть, и рассчитать меру перекрытия, рассматривая регулирующее устройство как устройство, задающее коэффициент местного сопротивления.

Цель работы: определить оптимальный способ закачивания в два трубопровода, в котором насос и трубопроводная сеть рассматриваются как единая взаимосвязанная система.

Объекты исследования: насосы, используемые для поддержания пластового давления, два трубопровода, снабжённых устройствами для регулирования расхода жидкости.

Методы исследования: метод для нахождения оптимального режима - вариационное исчисление.

Результаты. Работа насоса требует наименьшего количества энергии и времени, если заполнение заданных объёмов происходит одновременно. Для процесса экономичного закачивания в два трубопровода дан алгоритм определения степени перекрытия трубопроводов.

\section{Ключевые слова:}

Трубопровод, траектория закачки, насос, напор, подача, расход.

\section{Введение}

Для поддержания пластового давления (ППД) нефтяных месторождений используются высоконапорные насосы (более $10 \mathrm{MПа),} \mathrm{коэффициент}$ полезного действия (КПД) которых тем выше, чем больше подача (расход). Однако приемистость скважин (кубических метров в сутки) значительно уступает подаче насосов, что вынуждает использовать насосы для закачки жидкости через несколько трубопроводов в разные скважины [1-5]. Соответственно выбор наиболее эффективных режимов работы насосов становиться весьма актуальной за- 


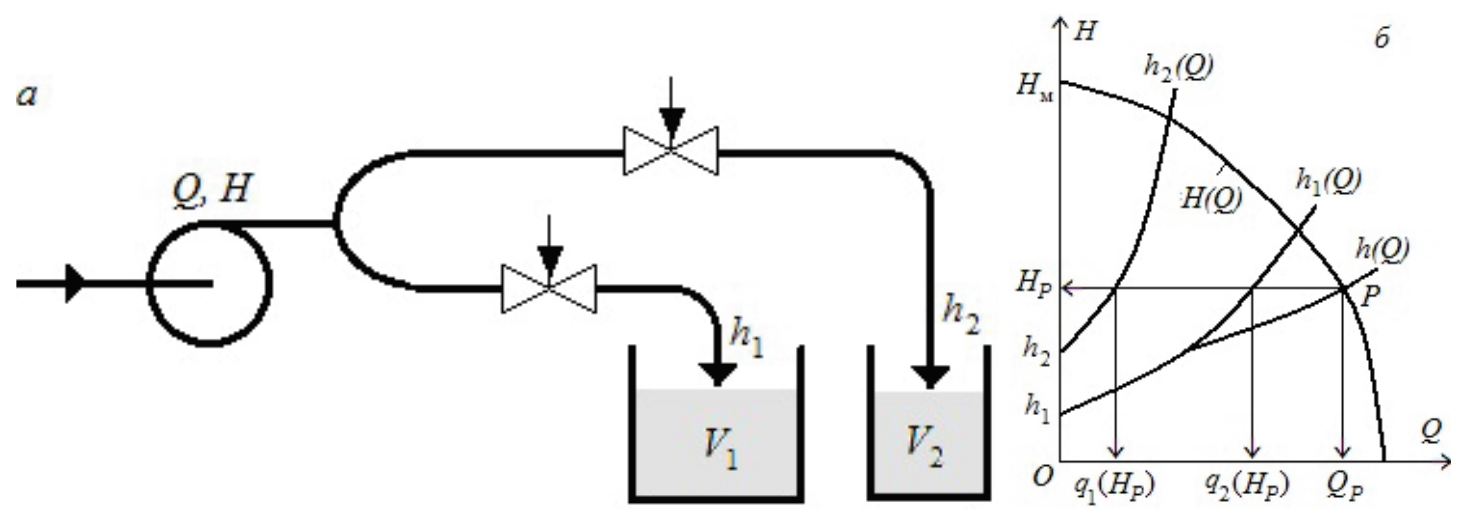

Pис. 1. Работа насоса на два трубопровода: а) схела закачки через два трубопровода; б) получение рабочей точки насоса

Fig. 1. Pump operation on two pipelines: a) injection scheme through two pipelines; б) obtaining the operating point of the pump

дачей, так как мощности насосных агрегатов, как правило, превышают 1 МВт,

В качестве критерия эффективности системы закачки используют затраты электрической энергии на единицу объёма перекачиваемой жидкости $[6,7]$, так называемый удельный расход электроэнергии (УРЭ), поскольку КПД насоса, не учитывает полное влияние параметров трубопроводной сети на количество затрачиваемой энергии [8-10].

\section{Постановка задачи}

Рассмотрим работу насоса на два трубопровода, снабжённых устройствами для регулирования расхода жидкости $[11,12]$. Через первый и второй трубопроводы требуется закачать объёмы $V_{1}$ и $V_{2}$ соответственно, затратив наименьшее количество энергии (рис. $1, a)$. Напорная характеристика наcoca $H(Q)$ и его КПД $\eta(Q)$ (где $Q$ - подача насоса; $H$ - напор, создаваемый насосом) обычно даются в виде графиков в паспорте насоса, поэтому они считаются известными. Пусть известны также гидравлические характеристики трубопроводов $\Delta h_{1}(Q)$, $\Delta h_{2}(Q)$, представляющие потери напоров в них, и соответствующие статические напоры $h_{1}, h_{2}$.

Потеря напора есть разность напоров в начальном и конечном сечениях трубы, поэтому

$$
H-h_{1}=\Delta h_{1}(Q), H-h_{2}=\Delta h_{2}(Q) .
$$

Отсюда

$$
H=h_{1}(Q), H=h_{2}(Q),
$$

где

$$
h_{1}(Q)=h_{1}+\Delta h_{1}(Q), h_{2}(Q)=h_{2}+\Delta h_{2}(Q) .
$$

На рис. 1, б трубопроводы представлены графиками функций $h_{1}(Q), h_{2}(Q)$. Поток $Q$, создаваемый насосом при напоре $H$, у входа в трубопроводы разделяется на потоки $q_{1}$ и $q_{2}$

$$
Q(H)=q_{1}(H)+q_{2}(H) .
$$

График правой части этого равенства изображён линией $h(Q)$ на рис. 1, б, получаемой сложением графиков $h_{1}(Q)$ и $h_{2}(Q)$ в горизонтальном направлении. Уравнение (1) определяет рабочую точку $P$ насоса с координатами $\left(Q_{p}, H_{P}\right)$ после чего становятся известными как расходы $q_{1}\left(H_{P}\right), q_{2}\left(H_{P}\right)$ в каждом трубопроводе, так и потребляемая насосом мощность

$$
N\left(Q_{P}\right)=\rho g \frac{H_{P} Q_{P}}{\eta\left(Q_{P}\right)},
$$

где $\rho$ - плотность перекачиваемой жидкости; $g$ ускорение силы тяжести.

В формуле (1) аргументом служит напор $H$. В большинстве случаев в целях простоты исследований в качестве независимой переменной будем принимать $H$. На графиках оси $H$ и $Q$ поменяются местами (рис. 2, a). (Если характеристика насоса содержит восходящую область, где работа насоса нестабильна, то соответствующий участок на оси $H$ исключается из рассмотрения; на остальных участках переменные $H$ и $Q$ связаны взаимно однозначным отношением.) При таком соглашении формула (2) принимает вид

$$
N\left(H_{P}\right)=\rho g \frac{H_{P} Q\left(H_{P}\right)}{\eta\left(H_{P}\right)} .
$$

\section{Степень влияния регулирующих устройств на характеристики потока}

Регулирующими устройствами уменьшим потоки в трубопроводах. Потери напора в устройствах увеличатся, гидравлические характеристики трубопроводов изменятся, и мы получим более низкие графики $\tilde{q}_{1}(H), \tilde{q}_{2}(H), \tilde{q}(H)$ (рис. $\left.2, \sigma\right)$. Рабочая точка опустится до положения $P \sim$ с координатами $\left(H_{\tilde{P}}, Q_{\tilde{P}}\right)$, определяемыми уравнением

$$
Q(H)=\tilde{q}_{1}(H)+\tilde{q}_{2}(H) .
$$

Факт того, что при увеличении перекрытия трубопроводов исходные графики $q_{1}(H), q_{2}(H)$ преобразуются в графики $\tilde{q}_{1}(H), \tilde{q}_{2}(H)$, которые всё более и более «прижимаются» $\kappa$ горизонтальной оси $H$, выразим соотношениями

$$
\tilde{q}_{1}(H)=a q_{1}(H), \quad \tilde{q}_{2}(H)=b q_{2}(H),
$$

в которых $a, b \in[0,1]$. Так, значению $a=0$ соответствует перекрытие первого трубопровода, а другому крайнему значению $a=1$ - его полная откры- 

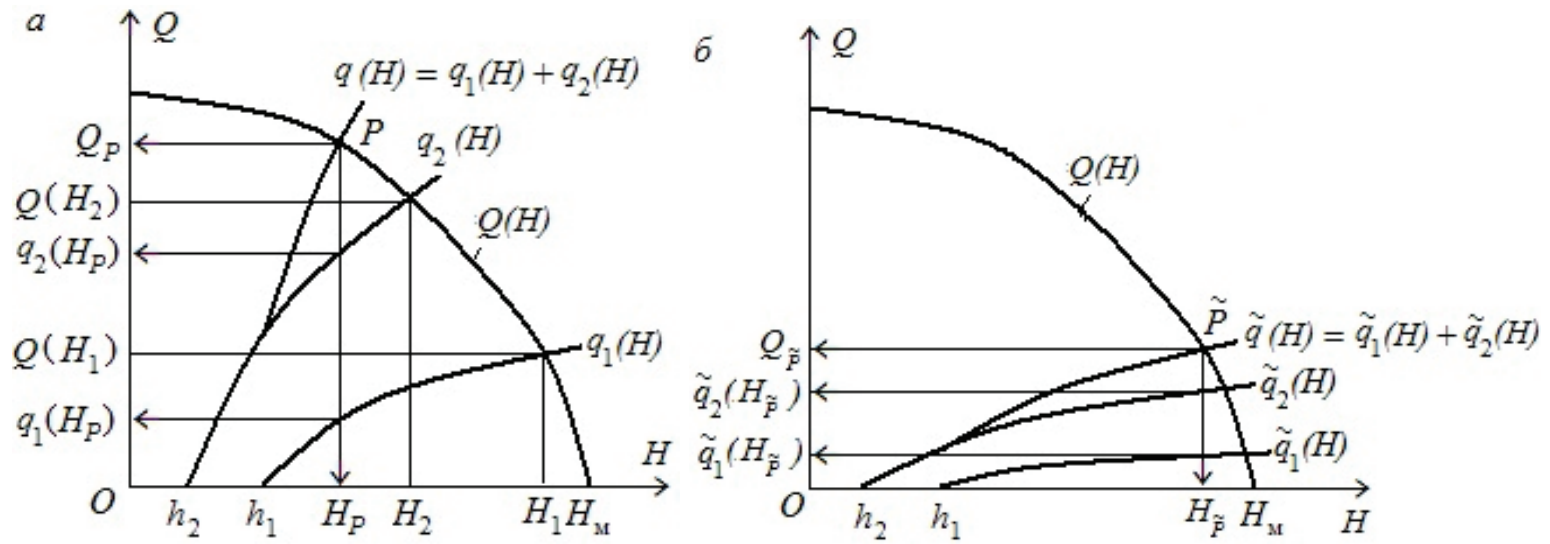

Pис. 2. Напорные характеристики насосов: а) сложение расходов $q_{1}(H), q_{2}(H)$; б) графики расходов и рабочей точки понизились в результате частичного перекрытия трубопроводов. $H_{\mathrm{M}}$ - наибольшее значение напора

Fig. 2. Pressure characteristics of pumps: a) addition of consumption; б) schedules of consumption and a working point have gone down as a result of partial overlapping of pipelines. $H_{\mathrm{M}}$ is the maximum head value

тость. Коэффициенты $a, b$ характеризуют степень открытости трубопроводов (или степень свободы потоков) и дают оценку влияния устройств на изменение потоков в трубопроводах. Подставив (4) в (3), получим уравнение, связывающие $a, b$ с расходами в трубопроводах:

$$
Q(H)=a q_{1}(H)+b q_{2}(H) .
$$

Из равенства $\tilde{q}_{1}(H)=a q_{1}(H)$ следует, что при уменьшении $a$ понижается график $\tilde{q}_{1}(H)$ и поэтому рабочая точка $\tilde{P}$ опускается - уменьшается $Q$ и увеличивается $H$. Следовательно,

при увеличении а или ь увеличивается общий поток $Q$ и уленьшается напор $H$.

Увеличим $a$, оставив $b$ неизменным. Согласно утверждению (А) увеличится $Q$ и уменьшится $H$. Уменьшение $H$ приводит к уменьшению значения $b q_{2}(H)$, т. к. $q_{2}(H)$ и $q_{1}(H)$ - возрастающие функции (рис. $2, a)$. Запишем (5) в виде

$$
a q_{1}(H)=Q(H)-b q_{2}(H) .
$$

Здесь правая часть возрастает, поэтому растёт и $a q_{1}(H)$. Таким образом,

увеличение а при постояннол $b$ приводит $\kappa$ росту $a q_{1}(H)$ и меньшению $b q_{2}(H)$.

\section{Введение функций, описывающих процесс закачки}

При закачке увеличиваются объёмы жидкости, поступившие через каждый трубопровод. Обозначим $x(t)$ и $y(t)$ объёмы, закачанные к моменту времени $t$ через первый и второй трубопроводы. Процесс закачки будет описываться уравнениями

$$
x=x(t), x=x(t),
$$

В начальный момент ни в один трубопровод ещё ничего не закачивалось:

$$
x(0)=0, y(0)=0 .
$$

$\kappa$ некоторому конечному моменту $T$ будут закачаны требуемые объёмы $V_{1}$ и $V_{2}$ :

$$
x(T)=V_{1}, y(T)=V_{2} .
$$

Равенства (7), (8) задают условия, которым должны удовлетворять функции $x(t), y(t)$. Отметим, что в равенствах (8) T есть момент окончания процесса закачки, который не обязан совпадать с моментом одновременного заполнения объёмов $V_{1}$ и $V_{2}$. Если объёмы $V_{1}$ и $V_{2}$ заполняются не одновременно, то $T$ есть момент заполнения последнего из них. В качестве примера рассмотрим случай, когда функции $x(t), y(t)$, описывающие процесс закачки (6), имеют вид, изображённый на рис. 3.

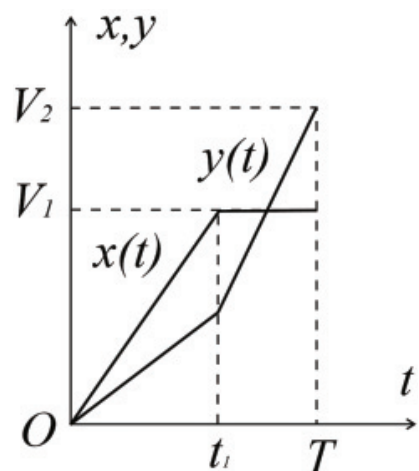

Рис.3. Графическое изображение возможного процесса неодновременного заполнения объёмов $V_{1} u V_{2}$

Fig. 3. Graphical representation of the possible non-simultaneous filling of volumes $V_{1}$ and $V_{2}$

Здесь мы видим, что в начальный период $\left[0, t_{1}\right]$ производится одновременная закачка в оба трубопровода. В момент времени $t_{1}$ первым заполняется объём $V_{1}$ и первый трубопровод перекрывается. После его перекрытия набранный объём $x(t)$ уже не меняется, оставаясь равным $V_{1}$, а поток во втором трубопроводе увеличивается, что отражено на рисунке увеличением крутизны графика $y(t)$ во 
второй период закачки $\left[t_{1}, T\right]$. В момент времени $T$ происходит окончательное заполнение объёма $V_{2}$. Рис. 3 показывает, что при $t=T$ имеем $x(T)=V_{1}$ и $y(T)=V_{2}$.

Равенства (6) задают параметрические уравнения линии на плоскости $x, y$ с переменным параметром $t$ (рис. $4, a$, б). Эту линию, изображающую процесс закачки, назовём траекторией закачки. Любая траектория закачки располагается в прямоугольнике $O V_{2} K V_{1}$, вершина $K$ которого соответствует времени $T$ и обозначает конечный момент закачки.
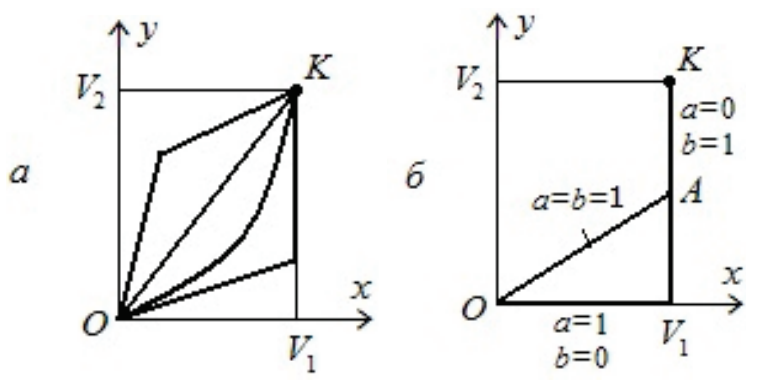

Pис.4. Траектории закачки: а) прилеры траекторий закачки; б) варианты закачки $O V_{1} K$ и OAK, выбранные для исследования их экономичности

Fig. 4. Injection trajectories: a) examples of injection paths; б) injection options $\mathrm{OV}_{1} \mathrm{~K}$ and $\mathrm{OAK}$ selected for studying their economy

Каждая точка прямоугольника изображает состояние процесса закачки. $\mathrm{K}$ примеру, точка с координатами $x=5, y=7$ может означать, что к определённому моменту времени через первый трубопровод закачали $5 \mathrm{~m}^{3}$ жидкости, а через второй $7 \mathrm{~m}^{3}$. Поэтому прямоугольник можно назвать фазовым пространством процесса закачки, точки в нём - фазовыми точками, линии - фазовыми траекториями. Насос вместе с трубопроводной сетью является динамической системой $[13,14]$.

\section{Зависимость потребляемой энергии от траектории закачки}

Убедимся в том, что потребляемая энергия зависит от траектории закачки $[15,16]$, сравнив значения энергии при закачивании по линиям $O V_{1} K$ и $O A K$ (рис. 4, б).

П е р в ы й случай: закачивание по траектории $O V_{1} K$. Сначала заполняется объём $V_{1}$ при перекрытом втором трубопроводе (процесс $O V_{1}$ на рис. 4 , б, при этом $a=1, b=0)$, затем заполняется $V_{2}$ при перекрытом первом трубопроводе (процесс $V_{1} K, a=0$, $b=1)$. В первый период рабочей точкой служит точка пересечения кривых $q_{1}(H)$ и $Q(H)$ (рис. 2, a). Время заполнения объёма $V_{1}$ равно $t_{1}=V_{1} / Q\left(H_{1}\right)$, при этом затрачивается энергия

$$
\varepsilon_{1}=N_{1} t_{1}=\rho g \frac{H_{1} Q\left(H_{1}\right)}{\eta\left(H_{1}\right)} t_{1}=\rho g \frac{H_{1} V_{1}}{\eta\left(H_{1}\right)} .
$$

При открытом втором трубопроводе получим

$$
\varepsilon_{2}=\rho g \frac{H_{2} V_{2}}{\eta\left(H_{2}\right)} \text {. }
$$

Суммарная потреблённая энергия равна

$$
E_{1}=\rho g \frac{H_{1} V_{1}}{\eta\left(H_{1}\right)}+\rho g \frac{H_{2} V_{2}}{\eta\left(H_{2}\right)} .
$$

В т о р о й случай: закачивание по траектории $O A K$. Сначала заполняются оба объёма до того момента, пока не заполнится один из них. Пусть первым заполнится объём $V_{1}$ (процесс $O A, a=1, b=1$ ), затем первый трубопровод перекрывается и продолжает заполняться объём $V_{2}$ (процесс $A K, a=0$, $b=1)$. В первый период, когда закачка производится через оба трубопровода, рабочей точкой будет пара чисел $\left(H_{P}, Q_{p}\right)$, определяемых уравнением (2) (рис. $2, a$ ). Так как первым заполняется объём $V_{1}$, то время одновременной закачки равно

$$
t_{1}=\frac{V_{1}}{q_{1}\left(H_{P}\right)},
$$

и в этот период будет затрачена энергия

$$
\varepsilon_{1}=\rho g \frac{H_{P} Q\left(H_{P}\right)}{\eta\left(H_{P}\right)} t_{1}=\rho g \frac{H_{P} Q\left(H_{P}\right) V_{1}}{q_{1}\left(H_{P}\right) \eta\left(H_{P}\right)} .
$$

В течение того же времени $t_{1}$ через второй трубопровод пройдёт объём $t_{1} q_{2}\left(H_{P}\right)$. В остальное время открытым будет только второй трубопровод $(a=0$, $b=1)$, где будет идти поток $Q\left(H_{2}\right)$ (рис. $\left.2, a\right)$. Поэтому оставшийся объём $V_{2}-t_{1} q_{2}\left(H_{P}\right)$ заполнится за время

$$
t_{2}=\frac{V_{2}-t_{1} q_{2}\left(H_{P}\right)}{Q\left(H_{2}\right)},
$$

и за это время будет потреблена энергия

$$
\varepsilon_{2}=\rho g \frac{H_{2} Q\left(H_{2}\right)}{\eta\left(H_{2}\right)} t_{2}=\rho g \frac{H_{2}\left[V_{2}-t_{1} q_{2}\left(H_{P}\right)\right]}{\eta\left(H_{2}\right)} .
$$

Общие затраты энергии равны

$$
E_{2}=\rho g \frac{H_{P} Q\left(H_{P}\right) V_{1}}{q_{1}\left(H_{P}\right) \eta\left(H_{P}\right)}+\rho g \frac{H_{2}\left[V_{2}-t_{1} q_{2}\left(H_{P}\right)\right]}{\eta\left(H_{2}\right)} .
$$

Для сравнения значений $E_{1}, E_{2}$ образуем их разность

$$
E_{1}-E_{2}=\rho g\left[\frac{H_{1} V_{1}}{\eta\left(H_{1}\right)}+\frac{H_{2} t_{1} q_{2}\left(H_{P}\right)}{\eta\left(H_{2}\right)}-\frac{H_{P} Q\left(H_{P}\right) V_{1}}{\eta\left(H_{P}\right) q_{1}\left(H_{P}\right)}\right] .
$$

Подставим значение $t_{1}$ из $(9)$ и $Q(H)=q_{1}(H)+q_{2}(H)$. После преобразований получим

$$
E_{1}-E_{2}=\frac{\rho g V_{1}}{q_{1}\left(H_{P}\right)}\left\{\begin{array}{l}
{\left[\frac{H_{1}}{\eta\left(H_{1}\right)}-\frac{H_{P}}{\eta\left(H_{P}\right)}\right] q_{1}\left(H_{P}\right)+} \\
+\left[\frac{H_{2}}{\eta\left(H_{2}\right)}-\frac{H_{P}}{\eta\left(H_{P}\right)}\right] q_{2}\left(H_{P}\right)
\end{array}\right\} .
$$

Рассмотрим поведение дроби $H / \eta(H)$, влияющей на выражения в квадратных скобках. Воспользуемся убыванием функции $H(Q)$ на интервале $\left(0, Q_{\mathrm{M}}\right)$ и выпуклостью функции $\eta(Q)$, имеющей максимум в точке $Q_{0}$ (рис. $5, a ; Q_{\mathrm{M}}$ - наибольшее возможное значение подачи). И докажем, что функция $\eta(H)$ тоже выпуклая. Для этого исследуем поведение величины 


$$
\eta^{\prime}(H)=\frac{\eta^{\prime}(Q)}{H^{\prime}(Q)} .
$$

Поскольку знаменатель отрицателен, функции $\eta^{\prime}(H), \eta^{\prime}(Q)$ имеют противоположные знаки. На интервале $\left(0, Q_{0}\right)$ имеем $\eta^{\prime}(Q)>0$, поэтому $\eta^{\prime}(H)<0$. Попутно отметим, что интервалу $\left(0, Q_{0}\right)$ оси $Q$ соответствует интервал $\left(H_{0}, H_{\mathrm{M}}\right)$ оси $H$. На следующем интервале $\left(Q_{0}, Q_{M}\right)$, которому соответствует интервал $\left(0, H_{0}\right)$, имеем $\eta^{\prime}(Q)<0$, поэтому $\eta^{\prime}(H)>0$. Получается, что при $H \in\left(0, H_{0}\right)$ дифференцируемая Это означает, что функция $\eta(H)$ выпуклая.

На рис. 5, б, где изображена выпуклая кривая $\eta(H)$, показано, что если $H_{1}<H_{2}$, то $\alpha_{1}>\alpha_{2}$, поэтому $\operatorname{ctg} \alpha_{1}>\operatorname{ctg} \alpha_{2}$ или $H_{1} / \eta(H)<H_{2} / \eta(H)$. Следовательно, переменные $H$ и $H / \eta(H)$ ведут себя одинаково, т. е. отношение

$$
\theta(H)=\frac{H}{\eta(H)}
$$

есть возрастающая функция напора $H$. В напорной характеристике насоса при увеличении $Q$ уменьшается $H$ (рис. 5, $a$ ). Поэтому аналогом величины (11) является функция

$$
\theta(Q)=\frac{H(Q)}{\eta(Q)},
$$

которая убывает при увеличении подачи $Q$.

В равенстве (10) $H_{1}>H_{p}, H_{2}>H_{p}$ (рис. 2, $a$ ), поэтому в квадратных скобках выражения положительны. Значит, $E_{1}>E_{2}$ - второй способ закачки экономичнее первого. Следовательно, не все траектории равнозначны по энергосбережению.

\section{Поиск траектории, на которой энергия минимальна}

Возьмём произвольную, но без изломов, траекторию $O K$, получаемую плавным регулированием устройствами во время закачки (рис. 5, в). Коэффициенты $a, b$ будут также плавно изменяться, а линия $O K$ опишется дифференцируемыми функциями $x(t), y(t)$. В этом случае существуют произфункция $\eta(H)$ растёт, а при $H \in\left(H_{0}, H_{\mathrm{M}}\right)$ убывает.

водные по времени $\dot{x} \equiv d x / d t, \dot{y} \equiv d y / d t$, означающие скорости закачки, или расходы жидкости в трубопроводах. Поэтому

$$
\dot{y}=b q_{2}(H),
$$

и формула (5) принимает вид

$$
Q(H)=\dot{x}+\dot{y} .
$$

За бесконечно малый промежуток времени $d t$ насос потребляет энергию $d E=N(Q) d t$. C учётом (13) её можно записать в виде $d E=N(\dot{x}+\dot{y}) d t$, поэтому за всё время $T$ работы он потребит энергию

$$
E=\int_{0}^{T} N(\dot{x}+\dot{y}) d t .
$$

Значение $E$ зависит от неизвестных функций $x(t)$ и $y(t)$, поэтому $E-$ функционал. Введём замену

$$
V=x+y \text {. }
$$

Равенства (7), (8) дают условия, которым должна удовлетворять функция $V(t)$ :

$$
V(0)=0, V(T)=V_{1}+V_{2} .
$$

Так как

$$
\dot{V}=\dot{x}+\dot{y},
$$

T0

$$
E=\int_{0}^{T} N(\dot{V}) d t .
$$

Входящая в эту формулу функция $V(t)$, как и $x(t), y(t)$, является гладкой, поэтому её можно найти с помощью уравнения Эйлера $[16,17]$ :

$$
\frac{d}{d t} N_{\dot{V}}=N_{V} .
$$

В силу того, что $N$ зависит лишь от $\dot{V}$, правая часть равна нулю. Остаётся $d N_{\dot{r}} / d t=0$, отсюда $N_{\ddot{W}} \ddot{V}=0$ Значит, $\ddot{V}=0$ или $N_{\ddot{W}}=0$. В обоих случаях решением является

$$
\begin{gathered}
\dot{V}=Q=\text { const } \\
V=Q t+C,
\end{gathered}
$$
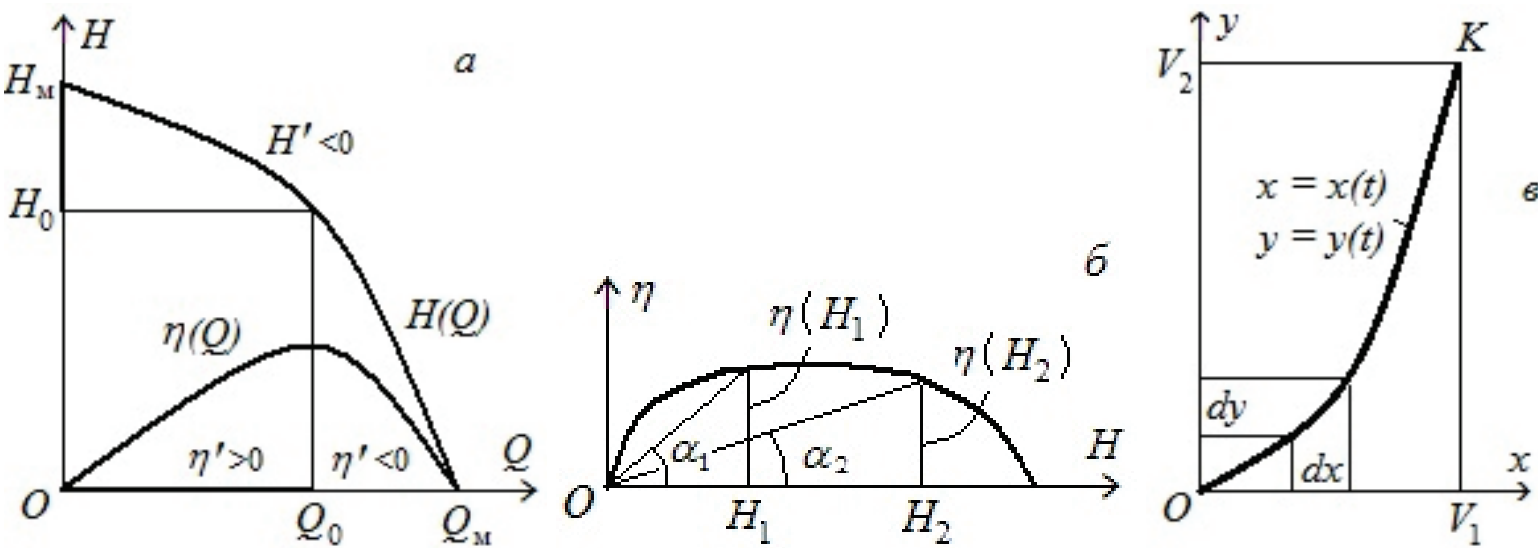

Pис. 5. Анализ поведения функиий: а) функиия $H(Q), \eta(Q)$ при доказательстве выпуклости $\eta(H)$; б) использование выпуклости $\eta(Н)$ для доказательства того, что функция $H / \eta(H)$ возрастающая; в) график произвольной траектории закачки без изломов

Fig. 5. Analysis of functions behavior: a) function $H(Q), \eta(Q)$ in the proof of convexity $\eta(H)$; $\sigma)$ use of convexity $\eta(H)$ to prove that the function $\mathrm{H} / \eta(\mathrm{H})$ is increasing; 8 ) graph of an arbitrary injection path without kinks 
где $C$ - постоянная [17]. Таким образом, подача насоса в процессе закачки не должна меняться. Подстановка условий (14) в (17) даёт время полной закачки

$$
T=\frac{V_{1}+V_{2}}{Q} .
$$

Из постоянства $Q$ следует постоянство характеристик $H, a, b$, а также мощности $N$ насоса. Поэтому выражение (15) сводится к равенству

$$
E=N T \text {. }
$$

С учётом условий (7) получаем решение уравнений (12):

$$
x=a q_{1}(H) t, \quad y=b q_{2}(H) t .
$$

Это уравнения прямой линии. Таким образом, траекторией закачки должен быть прямолинейный отрезок $O K$, изображающий одновременное заполнение обоих объёмов (рис. $4, a$ ).

\section{Определение значений коэффициентов открытости трубопроводов}

Подставим в (19) условия (8):

$$
a q_{1}(H) T=V_{1}, b q_{2}(H) T=V_{2} .
$$

Запишем выражение для энергии, подставив значение мощности:

$$
E=\rho g \frac{H}{\eta(H)} Q T .
$$

С учётом (18) получим

$$
E=\rho g \frac{H}{\eta(H)}\left(V_{1}+V_{2}\right) \text {. }
$$

Для минимизации $E$ нужно минимизировать величину $H / \eta(H)$, что достигается при наименьшем значении $H$. В соответствии с утверждением (A) мы можем уменьшить $H$, увеличив коэффициенты $a$ и $b$. Посмотрим, как можно найти подходящие коэффициенты.

Если из выражений (20) исключить $T$, получим связь между коэффициентами:

$$
\frac{a q_{1}(H)}{V_{1}}=\frac{b q_{2}(H)}{V_{2}} .
$$

Для того чтобы узнать, какой трубопровод не следует перекрывать, решим уравнение

$$
Q(H)=q_{1}(H)+q_{2}(H),
$$

получаемое из (5) при $a=b=1$, и найдём $H$. Это нетрудно сделать графическим способом. Найдя $H$, определим времена заполнения объёмов $V_{1}$ и $V_{2}$ :

$$
t_{1}=\frac{V_{1}}{q_{1}(H)}, t_{2}=\frac{V_{2}}{q_{2}(H)} .
$$

Пусть оказалось, что $t_{1}<t_{2}-$ первый объём заполняется быстрее второго. В таком случае, чтобы заполнить объёмы одновременно, нужно сдержать поток в первом трубопроводе, т. е. уменьшить $a$ и оставить по-прежнему $b=1$. В соответствии с (5) теперь мы должны иметь дело с уравнением

$$
Q(H)=a q_{1}(H)+q_{2}(H) .
$$

Привлечём сюда и соотношение (21), которое при $b=1$ даёт

$$
a=\frac{V_{1} q_{2}(H)}{V_{2} q_{1}(H)} .
$$

Подстановка этого выражения в предыдущее равенство приводит к уравнению

$$
Q(H)=\left(1+\frac{V_{1}}{V_{2}}\right) q_{2}(H),
$$

из которого найдём $H$, а затем $a$ из (22).

Если же $t_{1}>t_{2}$, то должно быть $a=1$ и мы получим уравнения

$$
Q(H)=\left(1+\frac{V_{2}}{V_{1}}\right) q_{1}(H), \quad b=\frac{V_{2} q_{1}(H)}{V_{1} q_{2}(H)},
$$

позволяющие определить $H$ и $b$ для этого случая.

\section{Закачка жидкости одним насосом}

\section{на три трубопровода}

В случае подключения насоса к трём трубопроводам аналогом уравнения (5) является уравнение

$$
Q(H)=a q_{1}(H)+b q_{2}(H)+c q_{3}(H)
$$

с коэффициентом $c$ открытости третьего трубопровода. В этом случае коэффициенты $a, b, c$ оказываются связанными равенствами

$$
\frac{a q_{1}(H)}{V_{1}}=\frac{b q_{2}(H)}{V_{2}}=\frac{c q_{3}(H)}{V_{3}} .
$$

Можно предложить следующий способ их определения. Решим, например, графически, уравнение

$$
Q(H)=q_{1}(H)+q_{2}(H)+q_{3}(H)
$$

и найдём $H$. Затем вычислим

$$
t_{1}=\frac{V_{1}}{q_{1}(H)}, t_{2}=\frac{V_{2}}{q_{2}(H)}, t_{3}=\frac{V_{3}}{q_{3}(H)} .
$$

Значения $t_{1}, t_{2}, t_{3}$ упорядочим по возрастанию. Например, так: $t_{1}<t_{2}<t_{3}-$ третий объём заполняется медленнее остальных. Тогда нужно взять $c=1$ и из (24) будем иметь

$$
a=\frac{V_{1} q_{3}(H)}{V_{3} q_{1}(H)}, b=\frac{V_{2} q_{3}(H)}{V_{3} q_{2}(H)} .
$$

Подстановка этих значений в (23) даст уравнение

$$
Q(H)=\left(1+\frac{V_{1}+V_{2}}{V_{3}}\right) q_{3}(H),
$$

из которого найдём $H$, затем $a, b$ из предыдущих формул.

\section{О траектории закачки, имеющей излом}

До сих пор предполагалось, что траектория закачки не имеет изломов. Проверим, возможна ли ломаная траектория, на которой энергопотребление будет ещё меньше.

Пусть $t_{0}$ - момент закачки, когда у траектории происходит излом. Время закачки разобьём на два 
интервала $\left[0, t_{0}\right]$ и $\left(t_{0}, T\right]$, в каждом из которых траектория закачки, а значит и функция $V(t)$, не имеет изломов. Поэтому мы можем применить уравнение Эйлера для каждого временного интервала отдельно, в результате чего придём к равенствам вида (16):

$$
\dot{V}=\left[\begin{array}{lll}
Q_{1} & \text { при } t \in\left[0, t_{0}\right] ; \\
Q_{2} & \text { при } t \in\left(t_{0}, T\right],
\end{array}\right.
$$

где $Q_{1}, Q_{2}$ - постоянные. При $t=t_{0}$ подынтегральная функция $N(\dot{V})$ в $(15)$ должна удовлетворять условиям Вейерштрасса-Эрдмана $[13,17]$ :

$$
\begin{aligned}
\left.N_{\dot{V}}\right|_{t=t_{0}-0} & =\left.N_{\dot{V}}\right|_{t=t_{0}+0}, \\
N-\left.\dot{V} N_{\dot{V}}\right|_{t=t_{0}-0} & =N-\left.\dot{V} N_{\dot{V}}\right|_{t=t_{0}+0} .
\end{aligned}
$$

В общем случае $N_{\dot{V}}$ есть нгепрерывная функция от $\dot{V}: N_{\dot{V}}=f(\dot{V})$ поэтому из первого условия следует

$$
\left.f(\dot{V})\right|_{t=t_{0}-0}=\left.f(\dot{V})\right|_{t=t_{0}+0} \text { или } f\left(Q_{1}\right)=f\left(Q_{2}\right),
$$

отсюда

$$
Q_{1}=Q_{2}
$$

- траектория закачки с наименьшим энергопотреблением не имеет изломов. Из непрерывности $N_{V}$ следует и выполнение второго условия Вейерштрасса-Эрдмана.

\section{Траектория, на которой время закачки минимально}

Пусть за время $d t$ через первый трубопровод закачался объём $d x$, через второй - объём $d y$ (рис. 5, в). В соответствии с (12)

$$
d x=a q_{1}(H) d t, d y=b q_{2}(H) d t .
$$

Отсюда

$$
\begin{gathered}
d x+d y=\left[a q_{1}(H)+b q_{2}(H)\right] d t=Q(H) d t, \\
d t=\frac{d x+d y}{Q(H)} .
\end{gathered}
$$

При фиксированных $d x, d y$ мы можем уменьшить время закачки $d t$, увеличив поток $Q(H)$. А увеличение потока достигается увеличением коэффициентов $a, b$. Однако их увеличение не может быть произвольным. В самом деле, из (25) следует

$$
\frac{b q_{2}(H)}{a q_{1}(H)}=\frac{d y}{d x} \equiv y^{\prime} .
$$

Так как $d x, d y$ у нас заданы, то правая часть постоянна. Попробуем увеличить $a$ при неизменном $b$. В соответствии с утверждением (Б) левая часть выражения (27) уменьшится. Поэтому, чтобы равенство (27) сохранилось, увеличение $a$ требует одновременного увеличения $b$. При их одновременном увеличении по крайней мере один из коэффициентов достигнет наибольшего значения, равного 1. Значит, чтобы на закачку ушло минимальное количество времени, на каждом участке траектории закачки либо $a$, либо $b$ должно быть равно 1 .

Из (27) и (5) составим равнозначные формулы

$$
Q(H)=a q_{1}(H)\left(1+y^{\prime}\right),
$$

$$
Q(H)=b q_{2}(H)\left(1+\frac{1}{y^{\prime}}\right) .
$$

Пусть на каком-то участке траектории закачки $a=1$. Из (28) получим

$$
Q(H)=q_{1}(H)\left(1+y^{\prime}\right) .
$$

Если же $b=1$, то из (29) будем иметь

$$
Q(H)=q_{2}(H)\left(1+\frac{1}{y^{\prime}}\right) .
$$

Последние две формулы говорят о том, что $H$ является функцией крутизны $y$ 'траектории закачки.

Так как $d x+d y=\left(1+y^{\prime}\right) d x$, где $x \in\left[0, V_{1}\right]$, то из (26) получим формулу вычисления времени закачки

$$
T=\int_{0}^{V_{1}} \frac{1+y^{\prime}}{Q(H)} d x .
$$

Для подынтегральной функции, зависимой лишь от $y^{\prime}$, введём обозначение

$$
F\left(y^{\prime}\right) \equiv \frac{1+y^{\prime}}{Q(H)} .
$$

Будем иметь функционал

$$
T=\int_{0}^{V_{1}} F\left(y^{\prime}\right) d x,
$$

значение которого зависит от $y(x)$. Таким же способом, которым определялись условия минимума энергии, получим $y^{\prime}=$ const - траектория закачки должна иметь постоянный угловой коэффициент, т. е. должна быть прямой. Значит, отрезок $O K$ есть траектория наименьших затрат времени на закачку.

\section{Расчёт степени открытия трубопроводов}

\section{с помощью коэффициентов открытости}

В гидравлических расчётах действие регулирующего устройства оценивают коэффициентом $\zeta$ местного сопротивления. Для турбулентного режима течения числовые значения $\zeta$ при различных степенях открытия трубопровода даются в справочных таблицах. Найдём связь между $\zeta$ и введенным нами коэффициентом открытости $a$.

Будем считать известным коэффициент $\zeta_{\text {труб }}$ сопротивления трубопровода при открытом устройстве, т. е. когда $a=1$. При $a=1$ потеря напора в трубопроводе равна

$$
\Delta h=\zeta_{\text {труб }} \frac{v^{2}}{2 g},
$$

где $v$ - скорость жидкости $[3,18]$. Так как $v=q / S$, где $S$ - площадь сечения трубы; $q$ - расход в трубопроводе,

$$
\Delta h=\frac{\zeta_{\text {труб }}}{2 g S^{2}} q^{2} .
$$

Отсюда

$$
q=\sqrt{\frac{2 g S^{2}}{\zeta_{\text {труб }}}} \sqrt{\Delta h} .
$$


Регулирующим устройством уменьшим расход, он станет равным $\tilde{q}$. Действие устройства увеличивает сопротивление трубопровода на некоторую величину $\zeta$, поэтому

$$
\tilde{q}=\sqrt{\frac{2 g S^{2}}{\zeta_{\text {труб }}+\zeta}} \sqrt{\Delta h} .
$$

Подставив значения $\tilde{q}, q$ в формулу, определяющую коэффициент открытости $a=\tilde{q} / q$, получим

$$
a=\sqrt{\frac{1}{1+\zeta / \zeta_{\text {труб }}},}
$$

отсюда

$$
\zeta=\left(\frac{1}{a^{2}}-1\right) \zeta_{\text {труб }}
$$

По найденному коэффициенту сопротивления задвижки $\zeta$ из соответствующих таблиц находим степень открытия трубопровода.

\section{Заключение}

Работа насоса требует минимума энергии и времени, если заполнение заданных объёмов происходит одновременно $[19,20]$. По результатам исследований можно предложить следующий алгоритм для осуществления наименее затратного процесса закачки в два трубопровода.

1. Имеем исходные данные: графики характеристик насоса $H(Q)$ и трубопроводов $h_{1}(Q)=h_{1}+\Delta h_{1}(Q)$, $h_{2}(Q)=h_{2}+\Delta h_{2}(Q)$ аналогичные тем, которые даны на рис. $1, \sigma$, где $\Delta h_{1}(Q), \Delta h_{2}(Q)$ - гидравлические характеристики трубопроводов, $h_{1}, h_{2}-$ статические напоры. Через первый и второй трубопроводы требуется закачать жидкость объёмов $V_{1}$ и $V_{2}$.

\section{СПИСОК ЛИТЕРАТУРЫ}

1. Бахтияров Г.А., Волков В.Г., Нургалиев Р.З. Опыт оптимизации работы системы поддержания пластового давления путем анализа текущей разработки нефтяного месторождения // Нефтепромысловое дело. - 2017. - № 3. - С. 9-15.

2. Parsasadr A., Ahmadi A., Keramat A. Waterhammer caused by intermittent pump failure in pipe systems including parallel puup groups // 37th IAHR World Congress 2017. - Kuala Lumpur, Malaysia, 2017. - P. 444-453.

3. Рабинович Е.3. Гидравлика. - М.: Недра, 1978. - 297 с.

4. Budxram D., Rasek M., Peters R. A simplified method for monitoring the efficiency of pumps // World Oil. - 2002. - № 6. P. 79-82.

5. Сулейманов Р.Н., Галеев А.С., Бикбулатова Г.И. Эффективность работы насосных агрегатов: монография. - Уфа: Изд-во УГНТУ, 2004. - $100 \mathrm{c}$.

6. Рахманов А.Р., Ожередов Е.В., Сафиуллин И.Р. Организация системы мониторинга энергоэффективности процессов добычи нефти // Нефтепромысловое дело. - 2017. - № 4. - С. 25-28.

7. Haghighi A., Keramat A. A fuzzy approach for considering uncertainty in transient analysis of pipe networks // Proc. of $11^{\text {th }} \mathrm{In}$ ternational Conference on Pressure Surges. - Lisbon, Portugal, 2012. - P. 24-26.
2. Меняем ролями оси и строим те же графики в системе координат $(H, Q)$. Получатся кривые $Q(H), q_{1}(H), q_{2}(H)$ подобные тем, что изображены на рис. $2, a$.

3. Графически решаем уравнение

$$
Q(H)=q_{1}(H)+q_{2}(H)
$$

и находим $H$. Далее определяем

$$
t_{1}=\frac{V_{1}}{q_{1}(H)}, t_{2}=\frac{V_{2}}{q_{2}(H)} .
$$

4. Если $t_{1}<t_{2}$, решаем уравнение

$$
Q(H)=\left(1+\frac{V_{1}}{V_{2}}\right) q_{2}(H),
$$

из него находим $H$ и вычисляем

$$
a=\frac{V_{1} q_{2}(H)}{V_{2} q_{1}(H)} .
$$

Теперь, зная $a$, перекрываем первый трубопровод с коэффициентом открытия $a$ и приступаем к закачке.

5. Если $t_{1}>t_{2}$, решаем уравнение

$$
Q(H)=\left(1+\frac{V_{2}}{V_{1}}\right) q_{1}(H),
$$

находим $H$ и вычисляем

$$
b=\frac{V_{2} q_{1}(H)}{V_{1} q_{2}(H)} .
$$

По $\zeta_{2}$ из таблиц находим степень открытия второго трубопровода. В соответствии с этим значением частично перекрываем второй трубопровод и начинаем закачку.

В следующей статье авторы планируют рассмотреть вопрос оптимизации параллельной работы двух и более насосов.

8. Методика определения КПд центробежных насосов системы поддержания пластового давления / А.Г. Богатырев, А.В. Лямин, Ю.А. Левин, И.Н. Елисеенко // Нефтепромысловое дело. - 2004. - № 5. - С. $22-25$.

9. Колосов Б., Сулейманов Р. Методика снижения энергоемкости процесса нагнетания в системе ППД и необходимое оборудование // Нефтесервис. - 2006. - № 3. - С. 27-29.

10. Методика определения КПД насосных агрегатов магистральных нефтепроводов // РД 39-0147103-307-85, Миннефтепром СССР, утвержден 14 декабря 1985. - Уфа: ВНИИСПТнефть, 1986. -36 c.

11. Shashi E. Menon Transmission Pipeline Calculations and Simulations Manual. - Oxford: ?Technology \& Engineering Publ., 2014. - 599 p.

12. Shashi E. Menon Pipeline Planning and Construction Field Manual. - 0xford: Technology \& Engineering Publ., 2011. - 552 p.

13. Анищенко В.С. Знакомство с нелинейной динамикой. - Саратов: СГУ, 2008. - 170 с.

14. Hanks K.H., Stanley G.T. Requirements of Pipeline Simulation: Accurately Modeling Transient Pipeline Operation // $19982^{\text {nd }}$ International Pipeline Conference. - Calgary, 1998. - V. II. P. 843-850.

15. Chemical Engineering. V. 1: Fluid Flow, Heat Transfer and Mass Transfer / J.F. Richardson, J.H. Harker, J.R. Backhurst et al. Oxford: Butterworth-Heinemann Title, 1999. - 526 p. 
16. Bird J. Engineering Mathematics. $5^{\text {th }}$ ed. - Amsterdam: Elsevier Ltd., 2007. -502 p.

17. Эльсгольц Л.Э. Дифференциальные уравнения и вариационное исчисление. - М.: Наука, 1969. - 425 c.

18. Milnes M. The Mathematics of Pumping Water. - Barnsley: AECOM Design Build, 2010. - 50 p.

19. Галеев А.С., Сулейманов Р.Н., Арсланов И.Г. Автономная система контроля и мониторинга работы насосной станции // Автоматизация, телемеханизация и связь в нефтяной промышленности. - 2013. - № 9. - С. 12-15.
20. Опыт применения автономной системы контроля и регистрации технологических параметров «АСКИР" / А.С. Галеев, Г.И. Бикбулатова, Ю.А. Болтнева, И.А. Кузьмин, П.П. Ермилов, А.О. Рязанцев // Нефтепромысловое дело. - 2016. № 9. - С. 43-48.

Поступила 29.05.2018 г.

\section{Информация об авторах}

Бикбулатова Г.И., кандидат технических наук, доцент кафедры нефтегазового оборудования и технологии машиностроения Альметьевского государственного нефтяного института.

Галеев A.C., доктор технических наук, профессор кафедры нефтегазового оборудования и технологии машиностроения Альметьевского государственного нефтяного института.

Болтнева Ю.А., старший преподаватель кафедры нефтегазового оборудования и технологии машиностроения Альметьевского государственного нефтяного института.

Ларин П.А., старший преподаватель кафедры информационных технологий, математики и естественных наук филиала Уфимского государственного нефтяного технического университета в г. Октябрьском.

Сулейманов P.Н., кандидат технических наук, доцент кафедры информационных технологий, математики и естественных наук филиала Уфимского государственного нефтяного технического университета в г. Октябрьском.

Филимонов О.В., кандидат технических наук, доцент кафедры информационных технологий, математики и естественных наук филиала Уфимского государственного нефтяного технического университета в г. Октябрьском. 
UDC 62-531.32

\section{OPTIMIZATION OF PUMPING FIXED VOLUME OF LIQUID ON TWO DIRECTIONS}

\section{Golia I. Bikbulatova',}

agni-ngo@mail.ru

\section{Achmetsalim S. Galeev',} agni-ngo@mail.ru

\section{Yulia A. Boltneva',} agni-ngo@mail.ru

Petr A. Larin, kafedra.itmen@mail.ru

Rais N. Suleymanov', kafedra.itmen@mail.ru

Oleg V. Filimonov ${ }^{2}$ kafedra.itmen@mail.ru

${ }^{1}$ Almetyevsk State Oil Institute,

2, Lenin street, Almetyevsk, Republic of Tatarstan, 423458, Russia.

2 Oktyabrsky branch of Ufa State Petroleum Technological University, 54 a, Devonskaya street, Oktyabrsky, Republic of Bashkortostan, 452620, Russia.

The paper introduces a new approach to solving the problem of optimization of dynamic systems "pump+pipe network». In the article the volume of fluid admitted through each pipe in a given time is called the state of pumping. These volumes are taken as basic time-dependent functions describing injection. The system of functions defines an imaginary line - the trajectory of injection in the space, set by the planned volumes of injection through each pipeline. Regulation of flow in the pipelines by any devices, which create local hydrodynamic resistance, allows obtaining different trajectories. The impact of control devices is estimated by the openness coefficient of a pipeline or flow, defined as the ratio of flow in partially blocked pipeline to the flow in the fully open pipeline. The paper considers two options of injection, which demonstrated that different trajectories could meet its own energy consumption. The authors have analyzed the value, equal to the ratio of the pressure produced by the pump to the efficiency corresponding to this pressure, used in quantitative research and assessment of pump operation. It is shown that with increasing pressure or with decreasing feed the given value grows. Since the required pump energy depends on the shape of the trajectory, the energy becomes a functional. To find the minimums of this functional the variational methods are used. This is the way for finding extremal trajectory, where energy and time cost is minimal. A trajectory describing the optimal injection mode is a straight-line segment in an injection space, the ends of which correspond to the initial and final states of injection. To implement this process, the authors proposed the algorithm to determine which pipeline should be partly blocked, and calculate a measure of blocking, considering control device as the device that sets the local resistance coefficient.

The main aim of the research was to determine the optimal way of pumping into two pipelines, in which the pump and the pipeline network are considered as a single interconnected system.

Objects: pumps used to maintain reservoir pressure, two pipelines equipped with devices for controlling fluid flow. Methods: method for finding the optimal regime - calculus of variations.

Results. Pump operation requires the least amount of energy and time, if the filling of the set volumes occurs simultaneously. The paper introduces the algorithm for determining the degree of blocking the pipelines for pumping in two pipelines.

Key words:

Pipeline, injection trajectory, pump, pressure, flow, consumption.

\section{REFERENCES}

1. Bakhtiyarov G.A., Volkov V.G., Nurgaliev R.Z. Experience of optimization of reservoir pressure maintenance system by analyzing current development of oil fields. Oilffield engineering, 2017 no. 3, pp. 9-15. In Rus.

2. Parsasadr A., Ahmadi A., Keramat A. Waterhammer caused by intermittent pump failure in pipe systems including parallel pump groups. $37^{\text {th }}$ IAHR World Congress 2017. Malaysia, Kuala Lumpur, 2017. pp. 444-453.

3. Rabinovich E.Z. Gidraulika [Hydraulics]. Moscow, Nedra Publ., 1978. $297 \mathrm{p}$

4. Budxram D., Rasek M., Peters R. Simplified method for monitoring the efficiency of pumps. World Oil, 2002, no. 6, pp. 79-82.
5. Suleymanov R.N., Galeev A.S., Bikbulatova G.I. Effectivnost raboti nasosnikh agregatov: monografia [Efficiency of pumping units operation: monograph]. Ufa, UGNTU Publ., 2004. 100 p.

6. Rakhmanov A.R., Ozheredov E.V., Safiullin I.R. Organization of the system providing monitoring of oil production energy efficiency. Oilffield engineering, 2017, no. 4, pp. 25-28. In Rus.

7. Haghighi A., Keramat A. A fuzzy approach for uncertainty in transient analysis of pipe networks. Proc. of the $11^{\text {th }}$ International Conference on Pressure Surges. Lisbon, Portugal, 2012. pp. 24-26.

8. Bogatyrev A.G., Lyamin A.V., Levin Yu.A., Yeliseyenko I.N. Method for determining the efficiency of centrifugal pumps of the reservoir pressure maintenance system. Oilffield engineering, 2004, no. 5. pp. 22-25. In Rus. 
9. Kolosov B., Suleimanov R. Technique for reducing the energy intensity of the injection process in the PPD system and the necessary equipment. Nefteservis, 2006, no. 3, pp. 27-29. In Rus.

10. Metodika opredeleniya KPD nasosnykh agregatov magistralnykh nefteprovodov [Methodology for determining the efficiency of pumping units of main oil pipelines]. RD 39-0147103-307-85, Minnefteprom USSR, approved on December 14, 1985. Ufa, VNIISPTneft Publ., 1986. 36 p.

11. Shashi E. Menon Transmission Pipeline Calculations and Simula tions Manual. 0xford, ?Technology \& Engineering Publ., 2014. $599 \mathrm{p}$.

12. Shashi E. Menon Pipeline Planning and Construction Field Manual. 0xford, Technology \& Engineering Publ., 2011. 552 p.

13. Anishchenko V.S. Znakomstvo s nelineinoy dinamikoy [Acquaintance with nonlinear dynamics]. Saratov, SSU Publ., 2008. 170 p.

14. Hanks K.H., Stanley G.T. Requirements of Pipeline Simulation: Accurately Modeling Transient Pipeline Operation. $19982^{n d} \mathrm{In}$ ternational Pipeline Conference. Calgary, 1998. Vol. II, pp. $843-850$.
15. Richardson J.F., Harker J.H., Backhurst J.R. Chemical Engineering. V. 1: Fluid Flow, Heat Transfer and Mass Transfer. 0xford, Butterworth-Heinemann Title, 1999. 526 p.

16. Bird J. Engineering Mathematics. $5^{\text {th }}$ ed. Amsterdam, Elsevier Ltd., 2007. $502 \mathrm{p}$.

17. Elsgolts L.E. Differentsialnye uravneniya i variatsionnoe ischislenie [Differential equations and calculus of variations]. Moscow, Nauka Publ., 1969. 425 p.

18. Milnes M. The Mathematics of Pumping Water. Barnsley, AECOM Design Build, 2010. $50 \mathrm{p}$.

19. Galeev A.C., Suleymanov R.N., Arslanov I.G. Autonomous system for monitoring the operation of the pumping station. Automation, telemechanization and communication in the oil industry, 2013, no. 9. pp. 12-15. In Rus.

20. Galeev A.S., Bikbulatova G.I., Boltneva Yu.A., Kuzmin I.A., Ermilov P.P., Ryazantsev A.O. Experience of application of «ASCIR" autonomous control systems and technological parameters registration. Oilffield engineering, 2016, no. 9, pp. 43-48. In Rus.

Received: 29 May 2018.

\section{Information about the authors}

Golia I. Bikbulatova, Cand. Sc., associate professor, Almetyevsk State Oil Institute.

Achmetsalim S. Galeev, Dr. Sc., professor, Almetyevsk State Oil Institute.

Yulia A.Boltneva, senior lector, Almetyevsk State Oil Institute.

Petr A. Larin, senior lector, Oktyabrsky branch of Ufa State Petroleum Technological University.

Rais N. Suleymanov, Cand. Sc., associate professor, Oktyabrsky branch of Ufa State Petroleum Technological University.

Oleg V. Filimonov, Cand. Sc., associate professor, Oktyabrsky branch of Ufa State Petroleum Technological University. 